\title{
Synergism Between Deca-brominated Flame Retardants and Nano-dispersed Clay in HIPS Composites
}

\author{
FEI YOU* ${ }^{1}$, YUAN HU ${ }^{1}$, YIING SHI ${ }^{2}$, and JIANJUN ZHOU ${ }^{1}$ \\ ${ }^{1}$ State Key Lab of Fire Science \\ University of Science and Technology of China \\ Jinzhai Road, No. 96, 230026 \\ Hefei, Anhui, China \\ ${ }^{2}$ Quality Inspection Station for Fire Fighting Products in Shandong Province \\ Shuitun Road, No. 59, 250033 \\ Jinan, Shandong, China
}

\begin{abstract}
High impact polystyrene (HIPS) composites containing both Decabromodiphenyl oxide (DBDPO) or 1, 2-bis (pentabromophenyl) ethane (DBDPE)-antimony (Sb2O3) and Montmorillonite (MMT, pristine clay)- Hexadecyl trimethyl ammonium bromide $(\mathrm{C} 16 \mathrm{BrN})$ flame retarding packages were prepared by one-step melt blending in one-pot. X-ray Diffraction (XRD), Transmission Electron Microscopy (TEM), Underwriters Laboratory Vertical Burning Test (UL $94 \mathrm{~V}$ ) and cone calorimeter tests have been performed to characterize the morphology and combustion properties of the flame retardant composites. Results show that HIPS-based nanocomposites with intercalation structure in the presence of conventional FR agent of DBDPO or DBDPE can be obtained, and much improved fire retardancy displayed by the notable reduction in heat release rates is achieved due to the good synergistic effect between MMT-C16BrN and DBDPO or DBDPE-antimony. Possible explanations regarding the synergism were proposed. This effect provides evidence for the reduction use of halogen-contained flame-retardants in one composite system for the purpose of lowering the production cost and lessening unwanted environment effects and is very meaningful for the development of green ecological polymer-based flame retardant composites.
\end{abstract}

KEYWORDS: high impact polystyrene, DBDPO-Antimony, DBDPE-Antimony, MMTC16BrN, nanocomposite, heat release rate, synergistic effect

\section{INTRODUCTION}

HIPS (High Impact Polystyrene) is a styrenic copolymer of polystyrene and grafted polybutadiene rubber. It is playing a very important role in the electronics industry because its widely uses for fabrication of TV or PC housings, household appliances and office equipments. However, HIPS resin and its end-use plastic products burn easily and pose a real fire threat and potential fire hazards to users. Therefore, it is a prerequisite to improve their resistance to fire before use. For television products, their fire retardancy must reach a certain level such as V-0 in USA and V-2 in Europe following the UL 94 standard [1]. This requirement can be achieved mainly by the use of flame retardant additives to the inexpensive and large volume resin. Brominated agents with antimony trioxide as a synergist are most often used for their high efficiency and relatively low cost. For its high bromine content as much as $83 \%$, decabromodiphenyl oxide (DBDPO) becomes the prevailing used brominated agent. Although it has been claimed by some European regulatory agencies that DBDPO-contained plastics release potential 
brominated dibenzo-p-dioxins and dibenzofurans (PBDD/F) in the process of recycling, landfiling, incinerating or burning and that DBDPO be replaced by counterparts or be banned, there indeed exist a wide range of data and practical experience demonstrating that the end-of-life management of products containing brominated flame retardants (BFRs) is fully compliable with an integrated waste management concept [2]. It is still advocated [3] that a market for BFRs be maintained through performance and the environmental arguments be solved to remove the restriction on the growth of BFRs. Presently, DBDPO still has a strong place in flame retardancy due to its unique property that a relatively small amount is enough to produce the required fire-retarding effect. Criterion that can be accepted for the applications of halogen-contained flame-retardants including DBDPO is that they can bring an effective fire retardancy, a retained or improved mechanical performance and a lessened negative ecological effect at the same time when added or incorporated in one system. Thane-1, 2-bis (pentabromophenyl) ethane (DBDPE), for its high efficiency and environment friendly characteristics, has become an alternative for DBDPO and gains more and more applications. Polymer/clay nanocomposites (PCN), the so-called "revolutionary" flame retarding system [4] recently developed, have been proved to bring both unique mechanical properties and a sustainable level of flame retardancy with $1 \% \sim 5 \%$ mass fractions of clay when compared to conventional filled polymers [5]. In this system, the clay phase can exist as either a delaminated (randomly dispersed silicate layers) or an intercalated (well order multiplayer with spacing between the silicate layers of only a few nanometers) [6] or the delamination/intercalation structure [7]. In this case, suppose to incorporate the conventional halogen-contained flame-retardants into a PCN system to investigate whether a good synergism exist between them. If does, the above performance requirements can be met especially for flame retardancy and mechanical properties. Further, a reduced amount of halogen-contained fire retardants or a lessened toxic effect can be expected in the newly called "fire retardant PCN". Previous researchers [8] have been exploring this synergistic effect in polypropylene layered silicate nanocomposites. In this paper, a 2:1 type clay mineral named montmorillonite (MMT) is applied to figure out its synergism with DBDPO or DBDPE in HIPS resin. Related combustion properties and morphological appearances were characterized.

\section{EXPERIMENTAL SECTION}

\section{Materials}

HIPS pellets with a trademark of HIPS 825TV provided by Fushun Petrochemical Complex of China have been used as the polymer composite matrix. Clay0, the purified sodium montmorillonite (Na+-MMT, China) with cation exchange capacity (CEC) of $96 \mathrm{meq} / 100 \mathrm{~g}$ and an interlayer spacing d001=1.5 $\mathrm{nm}$ has been used as the nanofiller precursor. It has been grounded into 200 meshes to better its dispersion into the polymer base. Hexadecyl trimethyl ammonium bromide (C16BrN), provided by Beijing Chemical Factory, has been selected as the clay/matrix reactive compatibilizer. DBDPOwith a bromine content of $83 \%$ provided by the Dead Sea Bromine Group, together with its synergistic agent of antimony trioxide $\left(\mathrm{Sb}_{2} \mathrm{O}_{3}\right)$ provided by Shenyang Huachang Antimony Industry Limited Company of China, has been used as added flame retardants. 


\section{The preparation of a Clay1}

In this paper, an organo-MMT with a mass ratio of 5 (Clay0) to 2 (C16BrN) was prepared by grinding them directly and manually in a mortar for 12 min to meet the simple one-step melt blending route. It is labeled as Clay1. XRD is measured to compare the $d$ (001) space with that of HIPS-clay composite to see whether polymer chains have been intercalated.

\section{The preparation of fire retardant HIPS composites}

All components involved (HIPS pellets, DBDPO, Sb2O3, MMT and C16BrN) were dried in a vacuum oven at $80^{\circ} \mathrm{C}$ for about 5 hours. Then throw them into a twin-roll mill (XK160 , made in Jiangsu China) at a time and keep them melt blended for $12 \mathrm{~min}$ at $180^{\circ} \mathrm{C}$ to yield fire retardant HIPS hybrids. Samples for cone calorimetric test were prepared by compressing the hybrids into a $10 \mathrm{~cm}$ x $10 \mathrm{~cm}$ square slab with a thickness of about $3 \mathrm{~mm}$ using a 1.00MN HPC-100 (D) Semi-automatic Moulding Press.

\section{Cone Calorimeter}

Heat release rate (HRR) was determined by the Stanton Redcroft cone calorimeter according to ISO 5660 under an external incident radiant flux set at $50 \mathrm{~kW} / \mathrm{m}^{2}$ using a cone-shaped heater. Samples were prepared with a dimension of $100 \mathrm{~cm}$ x $100 \mathrm{~cm}$ x $3 \mathrm{~mm}$. Post-combustion morphologies of samples were recorded using a type of CannonA 40 digital camera.

\section{UL-94 Test}

The test was carried out with samples of $13 \mathrm{~cm} \times 6.5 \mathrm{~mm} \times 3.0 \mathrm{~mm}$ each according to the American National Standard UL-94 (ANSI/ASTM D-635-77).

\section{Morphology of Clay in HIPS Resin Matrix}

The dispersibility of the MMT in the HIPS was evaluated using small-angle X-ray diffraction (XRD) and transmission electron microscopy (TEM). Thin films $(1 \mathrm{~mm})$ were pressed at $180^{\circ} \mathrm{C}$ for $\mathrm{XRD}$ measurements. X-ray diffraction tests were performed at room temperature with a Japan Rigaku D/max-rA X diffractometer (30 kV, $10 \mathrm{~mA}$ ) with $\mathrm{Cu} \mathrm{K}$ a irradiation $(\lambda=0.1505945 \mathrm{~nm})$ at the rate of $2^{\circ} / \mathrm{min}$ in the range of $1.5 \sim 10^{\circ}$. TEM specimens were placed on 200 mesh copper grids and measured by JEOL JEM-100SX with the acceleration voltage of $100 \mathrm{kV}$.

\section{RESULTS AND DISCUSSION}

Several fire retardant HIPS composites were prepared by "one-step melt blending in onepot" whose formulations are listed in Table 1. 
Table 1. Formulations of fire retardant HIPS composites.

\begin{tabular}{llllllll}
\hline \multirow{2}{*}{ Case } & \multicolumn{7}{c}{ Composition (Parts by weight, \%) } \\
\cline { 2 - 7 } & HIPS & DBDPO & DBDPE & $\mathbf{S b}_{2} \mathbf{O}_{3}$ & Clay0 & C16BrN & Br \\
\hline HIPS0 & 100 & & & & & & 0 \\
HIPS1a & 86 & 10 & & 4 & & & 8.30 \\
HIPS1b & 86 & & 10 & 4 & & & 8.23 \\
HIPS2 & 93 & & & & 5 & 2 & 0 \\
HIPS3 & 86 & & & & 10 & 4 & 0 \\
HIPS4a & 79 & \multirow{2}{*}{10} & & 4 & 5 & 2 & 8.30 \\
HIPS4b & 79 & & 10 & 4 & 5 & 2 & 8.23 \\
HIPS5 & 86 & 8 & & 2 & 3 & 1 & 6.64 \\
\hline
\end{tabular}

\section{Dispersion of MMT Layers in HIPS Resin}

XRD offers the opportunity to determine the spacing between MMT layers. In Clay0 the $\mathrm{d}$-spacing is $1.45 \mathrm{~nm}$. It can be increased if the sodium is ion-exchanged with an onium ion and further increased if polymer chains are incorporated into the gallery. Figure 1 shows the XRD patterns for Clay0, Clay1 and fire retardant HIPS composites, in which the first peaks correspond to the (001) plane reflections of MMT layers. Their characteristic values of d-spacing are listed in Table 2.

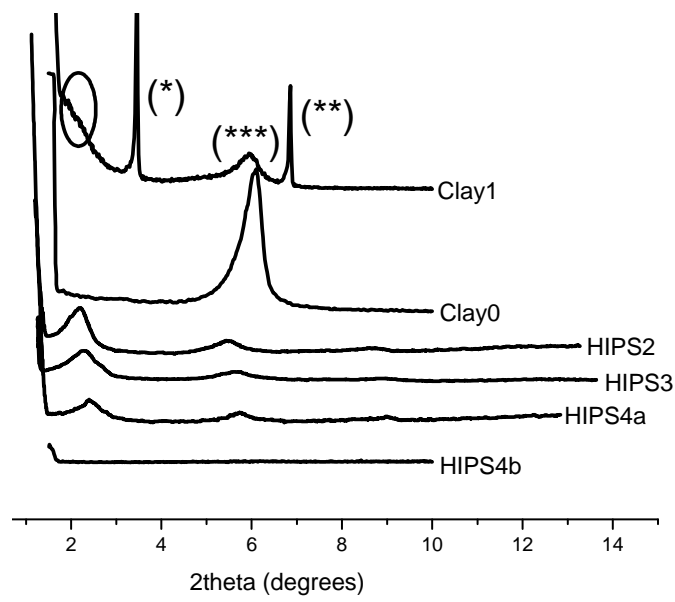

Fig.1. XRD patterns for clay0, clay1 and fire retardant HIPS composites.

As can be seen from Fig. 1, there is no clear peak for Clay1 at the angles from $1.5^{\circ} \sim 3^{\circ}$ . However, a strong tendency to peak is illustrated. The marked $(* * *)$ peak corresponds to a d-spacing of $1.48 \mathrm{~nm}$, indicating the unmodified MMT. This reflects that after mechanical grind MMT can be modified by $\mathrm{C} 16 \mathrm{BrN}$, but only a small portion of $\mathrm{C} 16 \mathrm{BrN}$ have been incorporated into the gallery. This is related to the current ambient moisture and grinding degree. As a result, the gallery height is broadened which is beneficial for the intercalation of polymer chains. The other two sharp peaks marked as $(*)$ and $\left({ }^{* *}\right)$ in the Clay1 XRD pattern corresponds to a d-spacing of $2.58 \mathrm{~nm}$ and $1.29 \mathrm{~nm}$, respectively, 
and have a second order diffraction relationship, indicating the typical diffraction peaks for C16BrN crystal. HIPS2, HIPS3 and HIPS4a have a d-spacing value of $3.85 \mathrm{~nm}$, $3.71 \mathrm{~nm}$ and $3.60 \mathrm{~nm}$, respectively. All of them are larger than that of pristine MMT and represent that HIPS chains are successfully interacted into the MMT layers. HIPS4b has no clear peak in small angels. TEM images are helpful to learn about the exact structure.

Table 2. XRD data for Clay0, Clay1 and fire retardant HIPS composites.

\begin{tabular}{lcc}
\hline & Case & $\mathbf{d}_{\mathbf{0 0 1}}$ space $(\mathbf{n m})$ \\
\hline Clay0 & 1.45 \\
Clay1 & (No clear peak) \\
HIPS2 & 3.85 \\
HIPS3 & 3.71 \\
HIPS4a & 3.60 \\
HIPS4b & -- \\
HIPS5 & -- \\
\hline
\end{tabular}

It is essential to have both XRD and TEM data to characterize a nanocomposite. The TEM images of HIPS4b, at both low and high magnification, are shown in Fig. 2 and Fig. 3. In Fig. 2, multi-layer clay tactoids can be seen. In Fig. 3, it can be clearly figured out that the nanocomposite is made up of finely dispersed multi-layer clay stacks. The gallery height calculated from the TEM image is $3.57 \mathrm{~nm}$, similar to that of HIPS4a shown in XRD. It can be safe to say that a HIPS/MMT nanocomposite with locally welldispersed intercalated structure has been achieved.

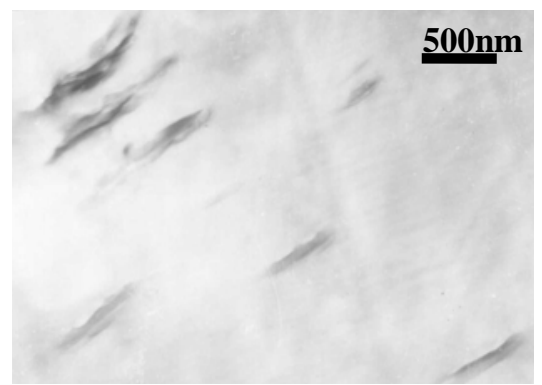

(a)

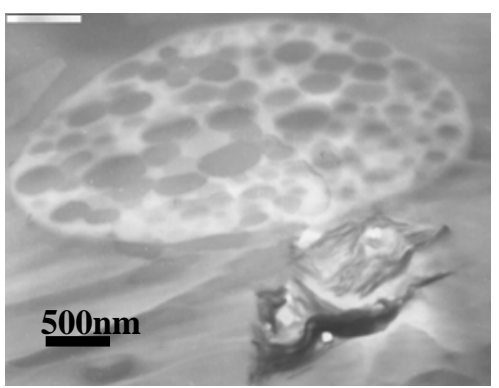

(b)

Fig. 2. Low magnification TEM of HIPS4b.

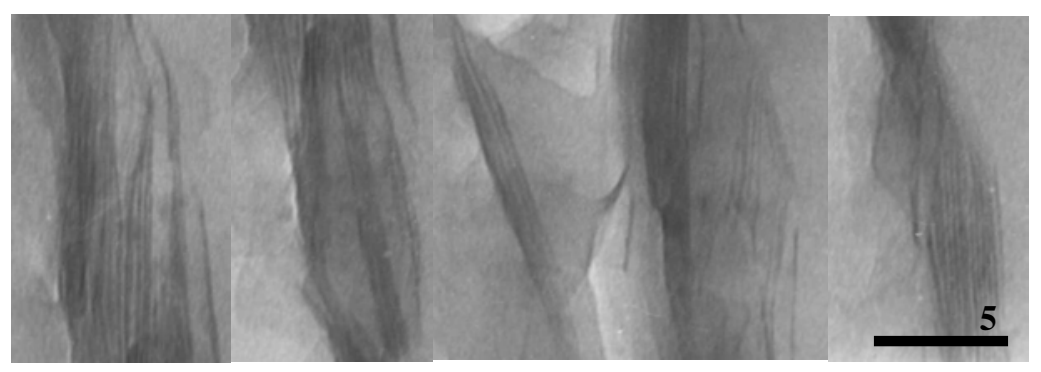

Fig. 3. High magnification TEM of HIPS4b (Intercalation). 


\section{Flammability Properties of Fire Retardant HIPS Composites}

\section{UL 94 Vertical Burning test results}

UL 94, "The Standard for Flammability of Plastic Materials for Parts in Devices and Appliances", determines a material's ability to propagate or extinguish a flame once ignited. The highest level is the V-0 rating that means "burning stops within 10 seconds without any drip" after the specimen has been exposed to a specified test flame under controlled laboratory conditions for 10 seconds. Results are listed in Table 3. As illustrated, pure HIPS resin and HIPS composites with only MMT-C16BrN keep burning after the flame has been removed, while HIPS1, HIPS4a, HIPS4b and HIPS5 selfextinguish immediately without any drip in 10 seconds. This indicates that DBDPOAntimony has high fire retarding efficiency; meanwhile, this effect is not impaired in the presence of MMT-C16BrN (HIPS4a, HIPS4b and HIPS5). Reversely, the combination of total amount of 14\% DBDPO-Antimony package (D-A) and 7\% MMT-C16BrN (M-C) package (HIPS4) guarantees the system to reach the stringent $\mathrm{V}-0$ rating. A further reduction of D-A by $3 \%$ and $\mathrm{M}-\mathrm{C}$ by $1.4 \%$ still keeps the $\mathrm{V}-0$ rating in HIPS5. These results show that when D-A and $\mathrm{M}-\mathrm{C}$ are used together, the overall combustion performances are not harmed. The relationship between D-A and M-C needs further qualitative studies.

Table 3. UL94 Rating and cone calorimeter data for fire retardant HIPS composites.

\begin{tabular}{|c|c|c|c|c|c|c|c|}
\hline \multirow{3}{*}{ Case } & \multirow{3}{*}{$\begin{array}{l}\text { UL } 94 \\
\text { Rating }\end{array}$} & \multicolumn{6}{|c|}{ Cone Calorimeter Data under heat flux of $50 \mathrm{~kW} / \mathrm{m}^{2}$} \\
\hline & & \multicolumn{2}{|c|}{$\begin{array}{l}\text { PkHRR@t } \\
\left(k W / m^{2} @ s\right)\end{array}$} & \multirow{2}{*}{$\begin{array}{l}\text { THR } \\
\left(\mathbf{M J} / \mathbf{m}^{2}\right)\end{array}$} & \multirow{2}{*}{$\begin{array}{l}\text { TTI } \\
\text { (s) }\end{array}$} & \multirow{2}{*}{$\begin{array}{l}\text { PkSEA@t } \\
\left(\mathrm{m}^{2} / \mathbf{k g} @ \mathbf{s}\right)\end{array}$} & \multirow{2}{*}{$\begin{array}{l}\text { PkEHC@t } \\
\text { (MJ/kg@s) }\end{array}$} \\
\hline & & Value & $\begin{array}{l}\text { Reduction } \\
\text { Rate (\%) }\end{array}$ & & & & \\
\hline HIPSO & Burning & $\begin{array}{l}1132.1 \\
9 @ 115\end{array}$ & 0 & 86.5 & 39 & 2051.73@155 & 64.98@140 \\
\hline HIPS1a & $V-0$ & $\begin{array}{l}581.01 \\
\text { @120 }\end{array}$ & -48.7 & 39.1 & 37 & 2987.83@155 & 36.45@135 \\
\hline HIPS1b & $\mathrm{V}-0$ & $\begin{array}{l}731.59 \\
@ 114\end{array}$ & -35.4 & 35.7 & 30 & 3137.67@117 & 80.00@114 \\
\hline HIPS2 & Burning & $\begin{array}{l}873.87 \\
\text { @80 }\end{array}$ & -22.8 & 63.9 & 28 & 2851.59@120 & 80.00@130 \\
\hline HIPS3 & Burning & $\begin{array}{l}768.75 \\
\text { @65 }\end{array}$ & -32.1 & 63.5 & 23 & 2805.02@135 & 76.88@150 \\
\hline HIPS4a & $V-0$ & $\begin{array}{l}403.35 \\
\text { @120 }\end{array}$ & -64.4 & 33.8 & 36 & 2994.13@100 & 36.92@135 \\
\hline HIPS4b & $\mathrm{V}-0$ & $\begin{array}{l}694.28 \\
@ 85\end{array}$ & -38.7 & 43.5 & 26 & 3015.54@115 & 80.00@130 \\
\hline HIPS5 & $\mathrm{V}-0$ & $\begin{array}{l}401.87 \\
\text { @93 }\end{array}$ & -64.5 & 36.6 & 26 & 2941.33@21 & 80.00@153 \\
\hline
\end{tabular}

\section{Cone Calorimeter Tests}

Cone calorimeter has long been used to characterize the bench-scale reaction-to-fire properties of materials. The peak HRR (PkHRR) is regarded as the single most important parameter to determine the fire hazard. Reduction rates of PkHRRs can be used to judge the effectiveness of fire retardancy.

Heat release profiles are shown in Fig. 4 and Fig. 5. Clearly seen from Fig. 4, DBDPOAntimony shows its high efficiency by only a sum amount of $14 \mathrm{wt} \%$ (HIPS1a) can reduce the peak HRR by $49 \%$. When MMT-C16BrN is used solely, an introduction of 
5\% clay0 and 2\% C16BrN (HIPS2) causes a reduction by 23\% in Peak HRR, a further addition of $10 \%$ clay0 and $4 \%$ C16BrN (HIPS3) reduces the peak HRR by $32 \%$. This result indicates that certain amounts of MMT-C16BrN can be used as flame retardant alone with a certain fire retarding effect. When both D-A and M-C with the same weight as HIPS1a and HIPS 2 are melted blended in one system (HIPS4a), another reduction of peak HRR by $16 \%$ comparing HIPS4a to HIPS1a. The results are in good compliance with UL 94 Ratings. This fully proves the synergistic effect of fire retardancy of D-A and M-C. It is predicted from this excellent synergistic effect that the combination between traditional flame-retardant of DBDPO-Antimony and nano-dispersed MMT-C16BrN can achieve equivalent fire ratings or improved fire properties using reduced additive amounts. The same conclusions can be made on DBDPE-Antimony and M-C for a combined 39\% reduction from original peak HRR of HIPS pure resin when both systems are included. However, this effect is not as notable as that in DBDPO-Antimony and M-C system.

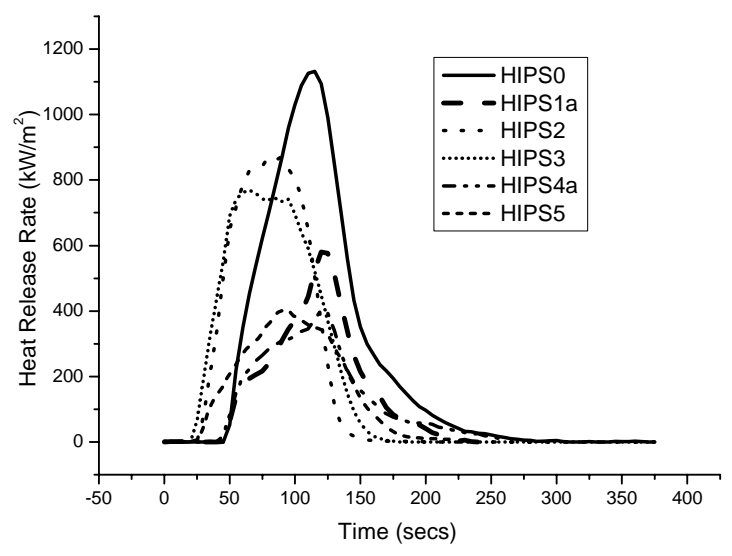

Fig. 4. HRR Profiles for DBDPO/Antimony retarded HIPS composites.

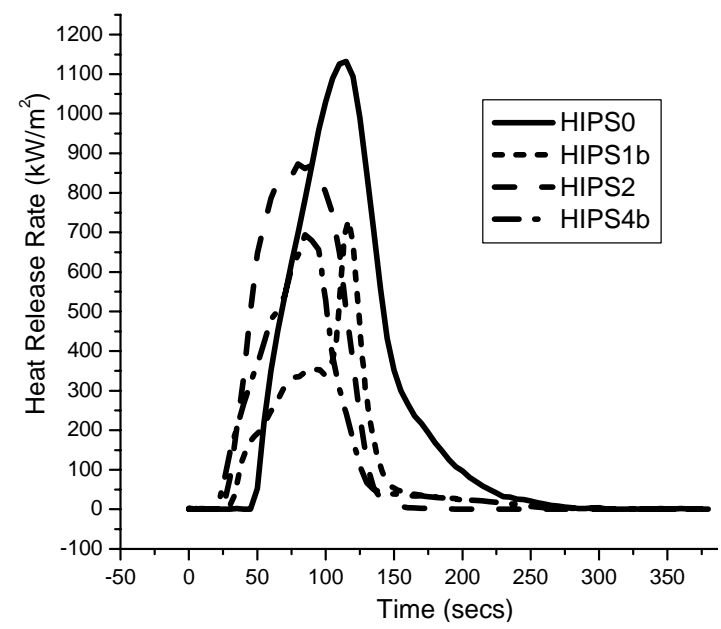

Fig. 5. HRR Profiles for DBDPE/Antimony retarded HIPS composites. 
Figures 6 and 7 show the THR (Total Heat Release) profiles. In Fig. 6, HIPS1a and HIPS2 display a reduction by $55 \%$ and $26 \%$ to that of HIPS0, respectively. For HIPS2 and HIPS3, an addition of M-C content does not change the THR values obviously. Their reduction ranges are only by $26 \%$ or so. These data indicate that $\mathrm{Br}$ has a larger role in reducing the total heat released. For HIPS4a, only a further reduction of $2 \%$ is achieved compared to that of HIPS1a. In Fig. 7, HIPS4b has a larger THR value than HIPS1b but a much lower THR value than HIPS2. The above results suggest that M-C package is not so effective in reducing total released heat as Br-Antimony package.

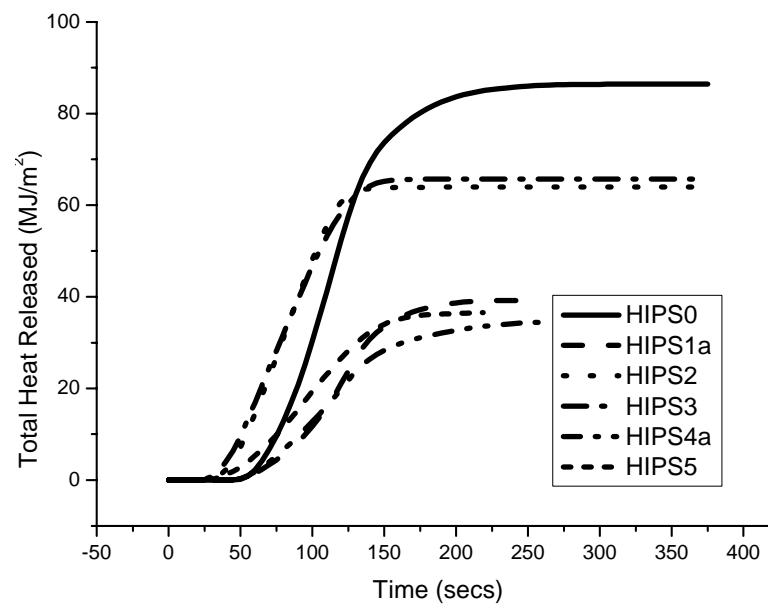

Fig. 6. THR Profiles for DBDPO/Antimony retarded HIPS composites.

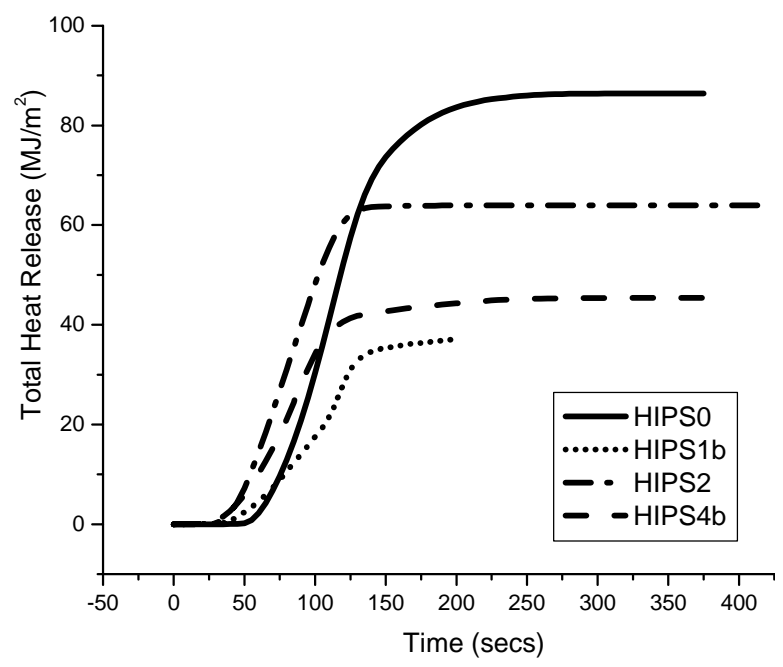

Fig. 7. THR Profiles for DBDPE/Antimony retarded HIPS composites.

As to the potential synergistic effect between two packages, possible explanations can be derived from the combustion of HIPS/Clay nanocomposites including deca-bromine type flame-retardants under piloted heat fluxes supplied in cone calorimeter. In the original HIPS resin, there disperse many components or units, such as free MMT particle and 
DBDPO, or intercalated MMT layers. When heated, the resin surface decomposes and releases volatile products including fragments and free radicals, this is the right time for $\mathrm{DBDPO} / \mathrm{Sb}_{2} \mathrm{O}_{3}$ play their role of trapping. A deoxidized atmosphere is provided. When the heat strengthens above the surface, the HIPS base melts and bubbles, clay aggregates or individual MMT layers or intercalated MMT units migrate to the surface by the role of buoyancy. Under the strong heat feedback from the gas phase flame, related clay structure will collapse and gathers at the surface of the molten polymer. A physical barrier is inclined to form. Meanwhile, fractured polymer dribs from the combustion chain terminated by brominated flame retardant, which provides "raw materials" under deoxidized atmosphere and stimulates the char formation. Finally, a thick and continuous carbonaceous char barrier may come into being to impede the transportation of volatiles and heat.

To sum up, two aspects regarding the synergistic effect can be proposed: first, free radical trapping and char barrier from $\mathrm{DBDPO} / \mathrm{Sb}_{2} \mathrm{O}_{3}$ and $\mathrm{MMT} / \mathrm{C} 16 \mathrm{BrN}$ take an active part in the earlier stage and later stage of pyrolysis and combustion, respectively; second, free radical trapping provides polymer fragments and deoxidized atmosphere for the later char formation by clay. Further studies are strongly required on more detailed and sound synergistic mechanism.

Typical residual chars after combustion from cone calorimeter tests are shown in Fig. 8.

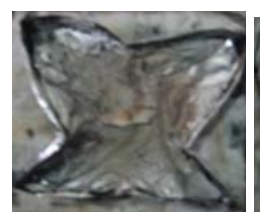

HIPS0

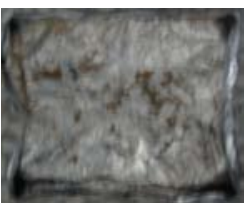

HIPS1

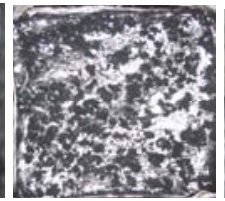

HIPS2

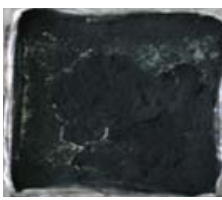

HIPS4a

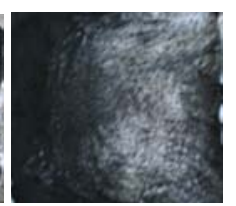

HIPS5

Fig. 8. Morphologies of combustion residues from cone calorimeter tests.

As can be seen clearly, HIPS resin (HIPS0) has been consumed completely without any solid residue left and an obvious deformation occurs in the burning process. The DBDPO-Antimony (HIPS1) impedes the combustion process and thus lessens heat feedback from the flame formed in the gas phase, the dimension is maintained and a few brown residues are formed. When MMT-C16BrN (HIPS2) is added, char formation capacity is improved and solid carbonaceous char residues are formed. However, they are scattered in small blocks. In HIPS4a and HIPS5, char formation capacity is greatly enhanced and a dense, tough and thick char residue is generated. It hinders the movement of volatilized polymer from the interior of a plastic matrix, denying fuel at the air/surface interface and abstain the much release of toxic products. However, due to the reduction of amounts used in HIPS5, char residues are locally condensed. Come what will, when both packages are used in HIPS resin, both fire retardancy and ecological performance are bettered, which meets the international tendency to develop materials with low toxicity, low heat, low smoke and balanced overall performances.

\section{CONCLUDING REMARKS}

By one-step melt blending conventional DBDPO-antimony and MMT-C16BrN with HIPS resin in one-pot, fire retardant HIPS nanocomposites can be obtained with both micro- (immiscible) and nano-dispersion (intercalated) morphologies. Comparing to HIPS composites with only DBDPO-Antimony or organo-MMT, fire retardant HIPS 
nanocomposites reach UL 94 ratings of V-0 easily and have further reduced peak HRR in cone calorimeter tests. In addition, char formation capacity has been clearly enhanced by MMT in the presence of DBDPO or DBDPE. Char residues after burning appear to be compacter and thicker. All of these are evidences for the good synergistic fire retarding effect between conventional DBDPO or DBDPE-Antimony and nano-dispersed organoMMT. Potential mechanism may lie in that the coupling between radical trapping in gas phase and barrier effect in condensed phase at earlier and later stage of combustion provides beneficial deoxidized atmosphere and fragments for the char formation which can interrupt the "Pyrolysis-Volatile-Combustion-Heat feedback" chain more effectively. As a result, more char residues and less volatiles are evolved. Taking advantage of this synergistic effect, the optimal ratio of MMT to DBDPO or DBDPE can be found by controlling DBDPO or DBDPE dosage through adjusting MMT amount. In this case, Polymer/MMT nanocomposites with low toxicity, high fire retardancy and optimized overall properties may be developed.

\section{ACKNOWLEDGEMENT}

The work was financially supported by the National Natural Science Foundation of China (No. 50476026) and the China NKBRSF project (No. 2001CB409600).

\section{REFERENCES}

[1] Kaspersmaa, J., Doumena, C., Munrob, S., and Prinsa, A.M., "Fire Retardant Mechanism of Aliphatic Bromine Compounds in Polystyrene and Polypropylene,” Polymer Degradation and Stability, 77, pp. 325-331, (2002).

[2] Tange, L., Drohmann, D., "Waste Management of Plastics Containing Brominated Flame Retardants," 2002 Conference on Flame Retardancy, London, United Kingdom, Feb. 5-6, 2002.

[3] Borms, R., "Making the Most of the Latest Technological Developments in Brominated Flame Retardants," Conference on flame retardants for electrical applications, Brussels, Belgium, March 5-6, 2003.

[4] Gilman, J.W., Kashiwagi, T., and Joseph, D.L., "Nanocomposites: A Revolutionary New Flame Retardant Approach," Proceedings of the 42nd International SAMPE Symposium, California, USA, 1997, pp. 1078-1089.

[5] Alexandre, M., and Dubois, P., "Polymer-layered Silicate Nanocomposites: Preparation, Properties and Uses of a New Class of Materials, Materials Science and Engineering," Reports: A review Journal 28, pp. 1-63, (2000).

[6] Kashiwagi T., Gilman, J.W., and Nyden M.R., "Polymer Combustion and New Flame Retardants," The 6th European Meeting on Fire Retardancy of Polymeric Materials (FRPM'97), Lille, France, Sept. 24-26, 1997, pp. 175-202.

[7] Gilman, J.W., Alex, M., Giannelis, E.P., Wuthenow, M., and Manias, E., "Flammability and Thermal Stability Studies of Polymer Layered-silicate (clay) Nanocomposites-II," Flame Retardancy, 10 th Annual BCC (Business Communications Co. Inc.) Conference, May 24-26, Stamford, CT, 1999, pp. 1-11.

[8] Zanetti, M., Camino, G., Canavese, D., Morgan, L.B., Lamelas, F.J., and Wilkie, C.A., "Fire Retardant Halogen-Antimony-clay Synergism in Polypropylene Layered Silicate Nanocomposites,” Chem. Mater, 14, pp. 189-193, (2002). 